\title{
Finite Dimensional Dynamics for Evolutionary Equations
}

\author{
Lychagin Valentin and Lychagina Olga \\ The University of Tromso
}

August 17, 2005

\begin{abstract}
We suggest a new method for investigation of finite dimensional dynamics for evolutionary differential equations and illustrate this method for the case of $\mathrm{KdV}$ equation. As a side result we give constructive solutions of a boundary problem for the Schrodinger equations which potentials are solutions of stationary $\mathrm{KdV}$ equations and their higher generalizations. ${ }^{1}$
\end{abstract}

Key words: evolutionary differential equations, shuffle symmetry, KdV equation, finite dimensional dynamic

\section{Introduction}

We suggest a method for investigation of finite dimensional dynamics for evolutionary differential equations and illustrate this method for the KdV equation. We outline the method for scalar evolutionary PDEs in dimension 2 but similar constructions for higher dimensional cases and systems of PDEs can be done in the same way by use results ([6]) instead of the classical Frobenius theorem.

Let's discuss in more details the case of scalar evolutionary PDEs in dimension 2. In this case there are two main points. First of all, if we consider an evolutionary PDE as a "dynamic" on a function space them a finite-dimensional sub-dynamic can be viewed as a dynamic on a solution space of some ordinary differential equation (ODE). This leads us to the second step of the description finite dynamics. We shall look for such ODEs that the given evolutionary PDE is a symmetry for the ODE. Putting all these together we can reformulate the problem of finding finite dimensional dynamics for an evolutionary PDE as a problem of finding ODEs for which the given evolutionary PDE is a symmetry. This gives us a differential equation for functions on jet spaces describing ordinary differential equations. In practice it is enough to find polynomial solutions of the equation.

\footnotetext{
${ }^{1}$ This manuscript has not been published or considered for publication elsewhere.
} 
The paper is organized as follows. In the first part we present geometrical theory of ODEs in the suitable for us form. Namely, we consider general ordinary differential equations (not only resolved with respect to highest derivative) and recall theory of shuffling symmetries and their use for integration ODEs. This type ordinary differential equations we'll need for description of dynamics. We illustrate this approach for the Schrödinger equation. This was done for two reasons: it is instructive to see how shuffling symmetries work for this case, and secondly, because these results shall be used in the second part in application to the $\mathrm{KdV}$ equations. It is worth to note that shuffle symmetries allow us to solve in quadratures the eigenvalue problem for the Schrödinger equations which potentials satisfy stationary KdV equation or its higher analogues. In the second part of the paper we describe in details low-dimensional dynamics (up to dimension 4) for KdV equation.

\section{ODEs and Dynamics}

\subsection{Geometry}

Denote by $\mathbf{J}^{m}$ the space of $m$-jets of scalar functions on $\mathbb{R}$ with canonical coordinates $\left(p_{m}, p_{m-1}, \ldots, p_{1}, p_{0}, x\right)$.

In these coordinates the Cartan distribution $\mathcal{C}_{m}$ on $\mathbf{J}^{m}$ ([1],[5]) given by the Cartan differential 1-forms

$$
\omega_{0}=d p_{0}-p_{1} d x, \cdots, \omega_{m-1}=d p_{m-1}-p_{m} d x .
$$

This is a 2-dimensional distribution and its generated by two vector fields

$$
\mathbf{D}_{m}=\frac{\partial}{\partial x}+p_{1} \frac{\partial}{\partial p_{0}}+\cdots+p_{m} \frac{\partial}{\partial p_{m-1}},
$$

and

$$
\frac{\partial}{\partial p_{m}}
$$

For any smooth function $f \in C^{\infty}\left(\mathbf{J}^{m}\right)$ we have

$$
d f=f_{p_{m}} d p_{m}+\mathbf{D}_{m}(f) d x \bmod \left(\omega_{0}, . ., \omega_{m-1}\right) .
$$

Define a bracket on the algebra $C^{\infty}\left(\mathbf{J}^{m}\right)$ as follows

$$
[f, g]=f_{p_{m}} \mathbf{D}_{m}(g)-g_{p_{m}} \mathbf{D}_{m}(f) .
$$

This is a skew-symmetric bracket which satisfies the following version of the Jacobi identity

$$
\begin{aligned}
& {[f,[g, h]]+[g,[h, f]]+[h,[f, g]]} \\
& =f_{p_{m-1}}[g, h]-g_{p_{m-1}}[f, h]+h_{p_{m-1}}[f, g] .
\end{aligned}
$$


The bracket is a bi-derivation and we denote by $Z_{f}$ the vector field corresponding to function $f$ :

$$
Z_{f}(g)=[f, g] .
$$

Then

$$
Z_{f}=\frac{\partial f}{\partial p_{m}} \mathbf{D}_{m}-\mathbf{D}_{m}(f) \frac{\partial}{\partial p_{m}} .
$$

Note that vector fields $Z_{f}$ belong to the Cartan distribution, and due to (2)

$$
\left[Z_{f}, Z_{g}\right]=Z_{[f, g]}+\frac{\partial f}{\partial p_{m-1}} Z_{g}-\frac{\partial g}{\partial p_{m-1}} Z_{f}+[f, g] \frac{\partial}{\partial p_{m-1}} .
$$

An ordinary differential equation (ODE)

$$
F\left(x, y, y^{\prime}, \ldots, y^{(m)}\right)=0
$$

of order $m$ in the standard way defines a subset

$$
\mathcal{E}=\left\{F\left(x, p_{0}, \ldots, p_{m}\right)=0\right\} \subset \mathbf{J}^{m} .
$$

We call point $x_{m} \in \mathcal{E}$ singular if either $\mathcal{E}$ is not smooth submanifold at $x_{m}$, or the tangent space $\mathbf{T}_{x_{m}} \mathcal{E}$ and the Cartan plane $\mathcal{C}_{m}\left(x_{m}\right)$ are not transversal, i.e.

$$
\mathcal{C}_{m}\left(x_{m}\right) \subset \mathbf{T}_{x_{m}} \mathcal{E}
$$

Denote by $\Sigma(\mathcal{E}) \subset \mathcal{E}$ the set of the singular points, and by $\mathcal{E}_{0}=\mathcal{E} \backslash \Sigma(\mathcal{E})$ the set of regular points of $\mathcal{E}$.

Note that the subset $\Sigma(\mathcal{E})$ of singular points is defined by equations

$$
F=0, \frac{\partial F}{\partial p_{m}}=0, \mathbf{D}_{m}(F)=0
$$

and in general has codimension 2 into $\mathcal{E}$.

The restriction of the Cartan distribution $\mathcal{C}_{m}$ on the regular part $\mathcal{E}_{0}$

$$
\mathcal{C}_{\mathcal{E}}: x_{m} \in \mathcal{E}_{0} \longmapsto \mathcal{C}_{\mathcal{E}}\left(x_{m}\right)=\mathcal{C}_{m}\left(x_{m}\right) \cap \mathbf{T}_{x_{m}} \mathcal{E}
$$

defines a 1-dimensional distribution on $\mathcal{E}_{0}$.

This is easy to see that this distribution is generated by the vector field $A_{F}$.

By solutions of ODE (4) we shall mean integral curves of the distribution $\mathcal{C}_{\mathcal{E}}$ or integral curves of $A_{F}$.

Note that condition $\frac{\partial F}{\partial p_{m}}\left(x_{m}\right) \neq 0$ at a point $x_{m} \in \mathcal{E}_{0}$ implies that coordinate function $x$ can be used as a local coordinate on the integral curve of $Z_{F}$ passing through the point, and therefore the curve can be presented at the form

$$
p_{m}=\frac{\partial^{m} h(x)}{\partial x^{m}}, \ldots . ., p_{0}=h(x)
$$

where the function $h(x)$ is the smooth solution of the ordinary differential equation (4).

If $\frac{\partial F}{\partial p_{m}}\left(x_{m}\right)=0$, then the smooth integral curve of $\mathcal{C}_{\mathcal{E}}$ shall represent a "multivalued" solution of (4). 
Example 1 The hypergeometric ordinary differential equation represents by function

$$
F=x(1-x) p_{2}+(c-(a+b+1) x) p_{1}-a b p_{0},
$$

where $a, b, c$ are constants with $a b \neq 0$. Then $\mathcal{E}$ is a 3 -dimensional submanifold in $\mathbf{J}^{2}=\mathbb{R}^{4}$ diffeomorphic to $\mathbb{R}^{3}$ and the singular set $\Sigma \mathcal{E}$ consists of two straight lines

$a b p_{0}+(1+a+b-c) p_{1}=0,(2+a+b-c) p_{2}+(1+a+b+a b) p_{1}=0, x=1$, and

$$
a b p_{0}-c p_{1}=0,(1+c) p_{2}-(1+a+b+a b) p_{1}=0, x=0 .
$$

Therefore integral curves which are not coincide with these two lines represent (multivalued) hypergeometric functions.

\subsection{Shuffle Symmetries}

By $\operatorname{Sol}(\mathcal{E})$ we denote the space of solutions of the ordinary differential equation $(4)$, that is, the set of all integral curves of the Cartan distribution $\mathcal{C}_{\mathcal{E}}$. In general this set does not possess any "good" topological or smooth structure, so we shall use geometry of jet spaces to induce a geometry on $\mathbf{S o l}(\mathcal{E})$. In some particular cases, for example when the equation can be resolved with respect to the highest derivative $F=p_{m}-F_{0}\left(x, p_{0}, \ldots, p_{m-1}\right), \mathcal{E} \approx \mathbb{R}^{m+1}$, and $\operatorname{Sol}(\mathcal{E}) \approx \mathbb{R}^{m}$. The last diffeomorphism can be done by taking the initial data.

Two notions have the greatest importance for us : functions and vector fields on $\operatorname{Sol}(\mathcal{E})$.

Namely, by functions on $\operatorname{Sol}(\mathcal{E})$ we understand the 1-st integrals of $\mathcal{E}$, or in other words functions $f$ on $\mathcal{E}$ which are smooth in some domain and constants on integral curves of $\mathcal{C}_{\mathcal{E}}: A_{F}(f)=0$,or

$$
[F, f]=0
$$

on $\mathcal{E}$.

"Vector fields" on $\mathbf{S o l}(\mathcal{E})$ correspond to (infinitesimal) symmetries of differential equation (4). One might consider symmetries as vector fields on $\mathcal{E}_{0}$ that are symmetries of the Cartan distribution $\mathcal{C}_{\mathcal{E}}$. It is easy to see that all vector fields proportional to $A_{F}$ are symmetries, and they are trivial (or characteristic) in the sense that they produce trivial ( or identity) transformations on the set $\operatorname{Sol}(\mathcal{E})$.

Because of the triviality we shall consider equivalence classes of symmetries modulo characteristic symmetries. We call them shuffle symmetries, (see [1]).

To find them we note that any such class has a representative $Y$ of the form

$$
Y=\sum_{i=0}^{m} a_{i} \frac{\partial}{\partial p_{i}} .
$$


Computing the Lie derivatives of the Cartan forms we get

$\mathbf{L}_{Y}\left(\omega_{j}\right)=d a_{j}-a_{j+1} d x=\frac{\partial a_{j}}{\partial p_{m}} d p_{m}+\left(\mathbf{D}_{m}\left(a_{j}\right)-a_{j+1}\right) d x \bmod \left(\omega_{0}, \ldots, \omega_{m-1}\right)=$

$$
\begin{aligned}
& \frac{-\frac{\partial a_{j}}{\partial p_{m}} \mathbf{D}_{m}(F)+F_{p_{m}}\left(\mathbf{D}_{m}\left(a_{j}\right)-a_{j+1}\right)}{F_{p_{m}}} d x \bmod \left(\omega_{0}, \ldots, \omega_{m-1}, d F\right) \\
& =\left(\frac{\left[F, a_{j}\right]}{F_{p_{m}}}-a_{j+1}\right) d x \bmod \left(\omega_{0}, \ldots, \omega_{m-1}, d F\right) .
\end{aligned}
$$

Because $d x$ does not vanish on $\mathcal{E}_{0}$ we get

$$
a_{j+1}=\delta\left(a_{j}\right) \text { on } \mathcal{E}_{0}
$$

for all $j=0, . ., m-1$.

Here

$$
\delta=\frac{1}{F_{p_{m}}} Z_{F} .
$$

Summarizing, we see that shuffling symmetries have representatives of the form

$$
X_{\phi}=\varphi \frac{\partial}{\partial p_{0}}+\delta(\varphi) \frac{\partial}{\partial p_{1}}+\ldots+\delta^{m}(\varphi) \frac{\partial}{\partial p_{m}}
$$

for some functions $\phi \in C^{\infty}\left(\mathcal{E}_{0}\right)$.

Requirement that $X_{\phi}$ tangents to $\mathcal{E}_{0}$ leads to the Lie equation

$$
\sum_{i=0}^{m} \frac{\partial F}{\partial p_{i}} \delta^{i}(\varphi)=0 \text { on } \mathcal{E}_{0}
$$

We call $\phi \in C^{\infty}\left(\mathcal{E}_{0}\right)$ generating functions of shuffling symmetries.

One can easily check that

$$
\left[X_{\phi}, X_{\psi}\right]=X_{[\phi, \psi]}
$$

where

$$
\begin{aligned}
& {[\phi, \psi]=X_{\phi}(\psi)-X_{\psi}(\phi)=} \\
& \sum_{i=0}^{m}\left(\frac{\partial \phi}{\partial p_{i}} \delta^{i}(\psi)-\frac{\partial \psi}{\partial p_{i}} \delta^{i}(\varphi)\right) .
\end{aligned}
$$

The bracket $[\phi, \psi]$ defines a Lie algebra structure on the space of all shuffling symmetries.

In order to see analytical meaning of the shuffling symmetry let us consider a smooth (low) solution of the ordinary differential equation $h_{0}(x)$ and let

$$
L_{0}=\left\{p_{0}=h_{0}, p_{1}=h_{0}^{\prime}, \ldots, p_{m}=h_{0}^{(m)}\right\}
$$

be the prolongation of $h_{0}(x)$ to $m$-jets. 
Let $A_{t}$ be the flow corresponding to a shuffling symmetry $X_{\varphi}$.

Then $L_{0} \subset \mathcal{E}$ and $L_{t}=A_{t}\left(L_{0}\right) \subset \mathcal{E}$.

Curves $\left\{L_{t}\right\}$ (at least locally and for small $t$ ) are $m$-jets of the functions $h_{t}(x)$, that is

$$
L_{t}=\left\{p_{0}=h_{t}, p_{1}=h_{t}^{\prime}, \ldots, p_{m}=h_{t}^{(m)}\right\}
$$

and $h_{t}(x)$ satisfies the same ordinary differential equation. Moreover, the function $u(t, x)=h_{t}(x)$ satisfies the following evolutionary equation

$$
\frac{\partial u}{\partial t}=\varphi\left(x, u, u_{x}, \ldots, \frac{\partial^{m} u}{\partial x^{m}}\right)
$$

and

$$
\left.u\right|_{t=0}=h_{0}(x) .
$$

In the other words, if $\varphi$ is a generating function of a symmetry and $h_{0}(x)$ is a solution of $\mathcal{E}$, then the function $u(t, x)$ satisfies equation (7) with initial data $u(0, x)=h_{0}(x)$, and $u(t, x)$ is a solution of $\mathcal{E}$ at any fixed moment $t$.

\subsection{Integration by symmetries}

We refer to ([1]) for integration of ordinary differential equations with solvable symmetry group, and discuss here the case of commutative symmetry algebra only.

We begin with the following observation. Let $v_{1}, . ., v_{n}$ be linear independent vectors fields on domain $D \subset \mathbb{R}^{n}$, and let

$$
\left[v_{i}, v_{j}\right]=0
$$

for all $i, j=1, \ldots, n$.

Take independent functions $f_{1}, . ., f_{n}$ on $D$ and define deferential 1-forms $\theta_{1}, \ldots, \theta_{n}$ as follows

$$
\theta=W^{-1} d f
$$

where

$$
\theta=\left[\begin{array}{c}
\theta_{1} \\
\theta_{2} \\
\vdots \\
\theta_{n}
\end{array}\right], d f=\left[\begin{array}{c}
d f_{1} \\
d f_{2} \\
\vdots \\
d f_{n}
\end{array}\right]
$$

and

$$
W=\left[\begin{array}{cccc}
v_{1}\left(f_{1}\right) & v_{2}\left(f_{1}\right) & \cdots & v_{n}\left(f_{1}\right) \\
v_{1}\left(f_{2}\right) & v_{2}\left(f_{2}\right) & \cdots & v_{n}\left(f_{2}\right) \\
\vdots & \vdots & \cdots & \vdots \\
v_{1}\left(f_{n}\right) & v_{2}\left(f_{n}\right) & \cdots & v_{n}\left(f_{n}\right)
\end{array}\right]
$$

Then $\theta_{1}, \ldots, \theta_{n}$ constitute the dual basis to $v_{1}, . ., v_{n}$.

Lemma $2 d \theta_{i}=0$ for all $i=1, \ldots n$. 
Proof. We have $\theta_{i}\left(v_{j}\right)=\delta_{i j}$, and therefore

$$
d \theta_{i}\left(v_{a}, v_{b}\right)=v_{a}\left(\theta_{i}\left(v_{b}\right)\right)-v_{b}\left(\theta_{i}\left(v_{a}\right)\right)-\theta_{i}\left(\left[v_{a}, v_{b}\right]\right)=0 .
$$

We apply this result for ordinary differential equation integration.

Assume that in a domain $\mathcal{D} \subset \mathcal{E}_{0} \subset \mathcal{E}=F^{-1}(0) \subset \mathbf{J}^{m}$ one has $m$ commuting linear independent shuffling symmetries $\phi_{1}, . ., \phi_{m}$. Then $\left[Z_{F}, X_{\phi_{i}}\right]=0$. Indeed, by the definition of symmetry $\left[Z_{F}, X_{\phi_{i}}\right]=\lambda Z_{F}$ for some function $\lambda$. Applying both sides to the coordinate function $x$ we get $\lambda=0$.

Therefore, vector fields $Z_{F}, X_{\phi_{1}}, \ldots, X_{\phi_{m}}$ commute and linear independent. To get 1-st integrals we need the following construction. Let us define a Cartan form $\omega_{f}$ corresponding to function $f \in C^{\infty}\left(\mathbf{J}^{m}\right)$ as follows

$$
\omega_{f}=\sum_{i=0}^{m} \frac{\partial f}{\partial p_{i}} \omega_{i}
$$

where

$$
\omega_{i}=d p_{i}-p_{i+1} d x, 0 \leq i \leq m-1,
$$

and

$$
\omega_{m}=\frac{F_{p_{m}} d p_{m}-\mathbf{D}_{m}(F) d x}{F_{p_{m}}} .
$$

Then

$$
\omega_{f}\left(X_{\phi}\right)=X_{\phi}(f) \quad \text { and } \quad \omega_{f}\left(Z_{F}\right)=0 .
$$

Theorem 3 Let $\phi_{1}, . ., \phi_{m}$ be commuting shuffling symmetries for ordinary differential equation $\mathcal{E}=F^{-1}(0) \subset \mathbf{J}^{m}$, and let $\mathcal{D} \subset \mathcal{E}_{0}$ be a domain where vector fields $X_{\phi_{1}}, \ldots, X_{\phi_{m}}$ are linear independent. Let $f_{1}, . ., f_{m}$ be functions such that functions $x, f_{1}, \ldots, f_{m}$ are independent in $\mathcal{D}$. Then differential 1 -forms $\theta_{1}, \ldots, \theta_{m}$ defined by

$$
\theta=W^{-1} \omega_{f}
$$

where

$$
\theta=\left[\begin{array}{c}
\theta_{1} \\
\theta_{2} \\
\vdots \\
\theta_{m}
\end{array}\right], \omega_{f}=\left[\begin{array}{c}
\omega_{f_{1}} \\
\omega_{2} \\
\vdots \\
\omega_{f_{m}}
\end{array}\right]
$$

and

$$
W=\left[\begin{array}{cccc}
X_{\phi_{1}}\left(f_{1}\right) & X_{\phi_{2}}\left(f_{1}\right) & \cdots & X_{\phi_{m}}\left(f_{1}\right) \\
X_{\phi_{1}}\left(f_{2}\right) & X_{\phi_{2}}\left(f_{2}\right) & \cdots & X_{\phi_{m}}\left(f_{2}\right) \\
\vdots & \vdots & \cdots & \vdots \\
X_{\phi_{1}}\left(f_{n}\right) & X_{\phi_{2}}\left(f_{n}\right) & \cdots & X_{\phi_{m}}\left(f_{m}\right)
\end{array}\right]
$$

are closed in $\mathcal{D}$ and $\theta_{i}\left(Z_{F}\right)=0$ for all $i=1, \ldots, m$. 
Proof. We have

$$
d x\left(Z_{f}\right)=1, \omega_{f_{i}}\left(Z_{f}\right)=0,
$$

and

$$
d x\left(X_{\phi_{i}}\right)=0, \omega_{f_{j}}\left(X_{\phi_{j}}\right)=X_{\phi_{j}}\left(f_{i}\right)
$$

for all $i, j=1, \ldots, m$.

Therefore differential 1-forms

$$
d x, \theta_{1}, \ldots ., \theta_{m}
$$

constitute the dual basis for

$$
Z_{F}, X_{\phi_{1}}, \ldots, X_{\phi_{m}}
$$

and the theorem follows from the above lemma.

Theorem 4 Let $\phi, \phi_{1}, . ., \phi_{r}$ be shuffling symmetries for ordinary differential equation $\mathcal{E}=F^{-1}(0) \subset \mathbf{J}^{m}$, and let $\mathcal{D} \subset \mathcal{E}_{0}$ be a domain where vector fields $X_{\phi_{1}}, \ldots, X_{\phi_{r}}$ are linear independent. If a vector field $X_{\phi}$ is a linear combination of the fields $X_{\phi_{1}}, \ldots, X_{\phi_{r}}$ then the shuffling symmetry $\phi$ is a linear combination of $\phi_{1}, . ., \phi_{r}$ in the domain $\mathcal{D}$ :

$$
\phi=\lambda_{1} \phi_{1}+\cdots+\lambda_{r} \phi_{r}
$$

where coefficients $\lambda_{1}, \ldots, \lambda_{r}$ are 1-st integrals for $\mathcal{E}$.

Proof. In the domain $\mathcal{D}$ we have

$$
X_{\phi}=\lambda_{1} X_{\phi_{1}}+\cdots+\lambda_{m} X_{\phi_{m}}
$$

for some functions $\lambda_{1}, \ldots, \lambda_{m}$.

Then $\phi=X_{\phi}\left(p_{0}\right)=\lambda_{1} \phi_{1}+\cdots+\lambda_{r} \phi_{r}$.

As we have seen $\left[Z_{F}, X_{\psi}\right]=0$, for all shuffling symmetries, therefore

$$
\left[Z_{F}, X_{\phi}\right]=Z_{F}\left(\lambda_{1}\right) X_{\phi_{1}}+\cdots+Z_{F}\left(\lambda_{m}\right) X_{\phi_{m}}=0
$$

and $Z_{F}\left(\lambda_{i}\right)=0$ for all $i=1, \ldots, m$.

\subsection{The Shrödinger equation}

\subsubsection{Linear Symmetries}

In this section we apply the above approach to the Shrödinger equation

$$
y^{\prime \prime}+w(x) y=0 .
$$

We look at the linear symmetries of this equation given by generating functions

$$
\phi=a(x) p_{1}+b(x) p_{0} .
$$


Substituting $\phi$ to the Lie equation we found that $\phi=c p_{0}+\phi_{z}$, where $c$ is a constant, and

$$
\phi_{z}=z(x) p_{1}-\frac{z^{\prime}(x)}{2} p_{0},
$$

where function $z=z(x)$ satisfies the following equation

$$
z^{\prime \prime \prime}+4 w z^{\prime}+2 w^{\prime} z=0 .
$$

Note that symmetries $p_{0}$ and $\phi_{z}$ commute and assuming that $z$ given one can find first integrals by quadratures. Namely, taking $f_{1}=p_{0}, f_{2}=p_{1}$ in theorem () one can get two differential 1-forms $\theta_{1}$ and $\theta_{2}$ and integrals $H_{1}$ and $H_{2}$.

The first integral $H=H_{1}$ can be chosen quadratic in $p_{0}, p_{1}$ :

$$
H=2 z p_{1}^{2}-2 z^{\prime} p_{0} p_{1}+\left(z^{\prime \prime}+2 w z\right) p_{0}^{2} .
$$

We'll rewrite this integral in the following way. Let us note that equation (9) is defined by the skew-adjoint operator

$$
L=\frac{d^{3}}{d x^{3}}+4 w \frac{d}{d x}+2 w^{\prime}
$$

and the Lagrange formula shows that

$$
K(z)=2 z\left(z^{\prime \prime}+2 w z\right)-z^{\prime 2}
$$

is a first integral for equation (9).

We say that symmetry $\phi_{z}$ is elliptic, hyperbolic or parabolic if $K(z)>0$, $K(z)<0$ or $K(z)=0$ respectively.

Using the symmetry we can rewrite the first integral in the form

$$
H=\frac{2\left(\phi_{z}^{2}+k p_{0}^{2}\right)}{z}
$$

where $4 k=K(z)$

Taking now $f_{1}=H, f_{2}=p_{0}$ in theorem () we find two differential 1-forms with

$$
\theta_{1}=\frac{d H}{2 H},
$$

and the restriction $\theta$ of the second form $\theta_{2}$ on levels $H=2 c$ equals to

$$
\theta=\frac{d p_{0}-p_{1} d x}{\phi_{z}}
$$

Let

$$
\begin{aligned}
& \alpha=\frac{\phi_{z}}{\sqrt{|z|}}, \\
& \beta=\frac{p_{0}}{\sqrt{|z|}} .
\end{aligned}
$$


Then

$$
H=2\left(\alpha^{2}+k \beta^{2}\right)=2 c
$$

and the restriction $\theta$ takes the following form

$$
\theta=\frac{d \beta}{\alpha}-\frac{d x}{z}
$$

Integration of $\theta$ gives the following solutions of the Schrödinger equation.

Theorem 5 Let $\phi_{z}$ be a linear symmetry of (8). Then solutions of the Shrödinger equation has the following form

- for elliptic symmetry $\phi_{z}$

$$
y(x)=\sqrt{\frac{|c z(x)|}{k}} \sin \left(\sqrt{k} \int \frac{d x}{z(x)}\right),
$$

- for hyperbolic symmetry $\phi_{z}$

$$
y(x)=\sqrt{\frac{|c z(x)|}{-k}} \sinh \left(\sqrt{-k} \int \frac{d x}{z(x)}\right),
$$

- for parabolic symmetry $\phi_{z}$

$$
y(x)=\sqrt{|c z(x)|} \int \frac{d x}{z(x)} .
$$

Here $H=2 c$.

\subsubsection{The Spectral Problem}

In this section we consider such potentials $w(x)$ that the corresponding eigenvalue problem

$$
y^{\prime \prime}+w(x) y=\lambda y
$$

possesses linear symmetries $z(x, \lambda)$ which are polynomial in $\lambda$.

Let

$$
z(x, \lambda)=z_{0}(x) \lambda^{n}+z_{1}(x) \lambda^{n-1}+\cdots+z_{n-1}(x) \lambda+z_{n}(x)
$$

be a linear symmetry for equation (10).

Then the Lie equation gives a polynomial (in $\lambda$ ) of degree $n+1$

$$
z^{\prime \prime \prime}(x, \lambda)+4(w(x)-\lambda) z^{\prime}(x, \lambda)+2 w^{\prime}(x) z(x, \lambda)=0
$$

and recursive set of equations on $z_{k}$ :

$$
\begin{aligned}
z_{0}^{\prime} & =0, \\
z_{k+1}^{\prime} & =\frac{1}{4} L\left(z_{k}\right), k=0, \ldots, n-1, \\
L\left(z_{n}\right) & =0 .
\end{aligned}
$$


Taking $z_{0}=1$, we get inductively functions $z_{k}=z_{k}(w)$ by

$$
z_{k+1}(w)=\frac{1}{4} \int L\left(z_{k}(w)\right) d x
$$

$k=0,1, \ldots$

The first functions are the following

$$
\begin{aligned}
z_{1}(w) & =\frac{w}{2}+c_{1}, \\
z_{2}(w) & =\frac{w^{\prime \prime}+3 w^{2}}{8}+\frac{c_{1}}{2} w+c_{2}, \\
z_{3}(w) & =\frac{w^{(4)}}{32}+\frac{5}{16}\left(w w^{\prime \prime}+\frac{w^{\prime 2}}{2}+w^{3}\right)+\frac{c_{1}}{8}\left(w^{\prime \prime}+3 w^{2}\right)+\frac{c_{2}}{2} w+c_{3}, \\
z_{4}(w) & =\frac{w^{(6)}}{128}+\frac{7 w^{\prime} w^{(3)}}{32}+\frac{7\left(3 w^{\prime \prime 2}+10 w^{2} w^{\prime \prime}+10 w w^{\prime 2}+5 w^{4}\right)}{128} \\
& +\frac{c_{1}}{32}\left(w^{(4)}+10\left(w w^{\prime \prime}+\frac{w^{\prime 2}}{2}+w^{3}\right)+\frac{c_{2}}{8}\left(w^{\prime \prime}+3 w^{2}\right)+\frac{c_{3}}{2} w+c_{4} .\right.
\end{aligned}
$$

The conditions $L\left(z_{n}(w)\right)=0$ which can be reformulated also as $z_{n+1}^{\prime}(w)=0$ are called $n$-th KdV stationary equations ([2]).

Below we list the first KdV equations :

0 -th $\mathrm{KdV}$

$$
w^{\prime}=0
$$

1-st KdV

$$
w^{\prime \prime \prime}+6 w w^{\prime}+4 c_{1} w^{\prime}=0
$$

2-nd $\mathrm{KdV}$

$$
w^{(5)}+10\left(w w^{\prime \prime \prime}+2 w^{\prime} w^{\prime \prime}+3 w^{2} w^{\prime}\right)+4 c_{1}\left(w^{\prime \prime}+6 w w^{\prime}\right)+16 c_{2} w^{\prime}=0 .
$$

We conclude that potentials $w$ which satisfy the $n$-th stationary KdV equation possess linear symmetry $\phi_{S_{n}}$ with

$$
S_{n}=\lambda^{n}+\sum_{k=1}^{n} z_{k} \lambda^{n-k} .
$$

As we have seen function $K=2 z\left(z^{\prime \prime}+2(w-\lambda) z\right)-z^{2}$ is the first integral of the Lie equation and therefore coefficients of the polynomials

$$
Q_{n}=2 S_{n}\left(S_{n}^{\prime \prime}+2(w-\lambda) S_{n}\right)-S_{n}^{2}
$$

are first integrals for the $n$-th $\mathrm{KdV}$ equation.

For example, for the classical (first) $\mathrm{KdV}$ equation, $w^{\prime \prime \prime}+6 w w^{\prime}+4 c_{1} w^{\prime}=0$, one has

$$
S_{1}=\lambda+\frac{w}{2}+c_{1}
$$


and

$$
Q_{1}=-4 \lambda^{3}-8 c_{1} \lambda^{2}+q_{11} \lambda+q_{10}
$$

where

$$
\begin{aligned}
& q_{11}=w^{\prime \prime}+3 w^{2}+4 c_{1} w-4 c_{1}^{2}, \\
& q_{10}=\frac{2 w w^{\prime \prime}-w^{\prime 2}+4 w^{3}}{4}+c_{1}\left(w^{\prime \prime}+4 w^{2}+4 c_{1} w\right)
\end{aligned}
$$

are first integrals.

Solving the KdV equation together with equations $q_{11}=$ const, $q_{10}=$ const we get 1 -st order ODE for $w$ :

$$
w^{\prime 2}=-2 w^{3}-4 c_{1} w^{2}+2\left(q_{11}+4 c_{1}^{2}\right) w+4\left(c_{1} q_{11}-q_{10}+4 c_{1}^{3}\right)
$$

and solutions

$$
w=-2 \wp\left(x+c, g_{2}, g_{3}\right)-\frac{2 c_{1}}{3}
$$

where $\wp\left(x, g_{2}, g_{3}\right)$ is the Weierstrass elliptic function with invariants

$$
g_{2}=\frac{4 c_{1}^{2}-6 c_{1}}{3}, g_{3}=-\frac{152 c_{1}^{3}}{27}-\frac{5 q_{11} c_{1}}{3}+2 q_{10} .
$$

For the second $\mathrm{KdV}$ equation,

$$
w^{(5)}+10\left(w w^{\prime \prime \prime}+2 w^{\prime} w^{\prime \prime}+3 w^{2} w^{\prime}\right)+4 c_{1}\left(w^{\prime \prime}+6 w w^{\prime}\right)+16 c_{2} w^{\prime}=0,
$$

one has

$$
S_{2}=\lambda^{2}+\left(\frac{w}{2}+c_{1}\right) \lambda+\frac{w^{\prime \prime}+3 w^{2}}{8}+\frac{c_{1}}{2} w+c_{2}
$$

and

$$
Q_{2}=-4 \lambda^{5}-8 c_{1} \lambda^{4}-4\left(c_{1}^{2}+2 c_{2}\right) \lambda^{3}+q_{22} \lambda^{2}+q_{21} \lambda+q_{20}
$$

where

$$
\begin{aligned}
q_{22} & =\frac{10 w^{3}+5 w^{\prime 2}+10 w w^{\prime \prime}+w^{(4)}}{10}-8 c_{1} c_{2}+\left(w^{\prime \prime}+3 w^{2}\right) c_{1}+4 w c_{2}, \\
q_{21} & =\frac{2 w w^{(4)}-2 w^{\prime} w^{\prime \prime \prime}+w^{\prime \prime 2}+20 w^{2} w^{\prime \prime}+15 w^{4}}{16}+\left(w^{\prime \prime}+3 w^{2}\right) c_{1}^{2}+4 w c_{1} c_{2}-4 c_{2}^{2}+ \\
& \frac{4 w^{(4)}+12 w w^{\prime \prime}+4 w^{\prime 2}+14 w^{3}}{4} c_{1}+w^{2} c_{2}, \\
q_{20} & =\frac{2 w^{\prime \prime} w^{(4)}+6 w^{2} w^{(4)}+4 w\left(4 w^{\prime \prime 2}-3 w^{\prime} w^{\prime \prime \prime}\right)-w^{\prime \prime \prime}+12 w^{\prime 2} w^{\prime \prime}+60 w^{3} w^{\prime \prime}+36 w^{5}}{64}+ \\
& \frac{4 w^{3}-w^{\prime 2}+2 w w^{\prime \prime}}{4} c_{1}^{2}+\left(w^{\prime \prime}+4 w^{2}\right) c_{1} c_{2}+4 w c_{2}^{2}+ \\
& \frac{12 w^{4}+13 w^{2} w^{\prime \prime}+w^{\prime \prime 2}-w^{\prime} w^{\prime \prime \prime}+w w^{(4)}}{8} c_{1}+\frac{12 w^{3}+6 w^{\prime 2}+10 w w^{\prime \prime}+w^{(4)}}{4} c_{2}
\end{aligned}
$$

are the first integrals for the second KdV. 
Using these integrals one can reduce the 2 -nd $\mathrm{KdV}$ equation to the following 2-nd order ODE $\mathcal{E}_{J T}$ :

$$
\begin{aligned}
& 25 w^{8}+80 w^{7} c_{1}+32 w^{6}\left(2 c_{1}^{2}+5 c_{2}\right)-16 w^{5}\left(24 c_{1} c_{2}+5 Q_{22}\right)+ \\
& 32 w^{4}\left(-72 c_{1}^{2} c_{2}+28 c_{2}^{2}+5 Q_{21}-9 c_{1} Q_{22}\right)+256\left(-8 c_{1}^{2} c_{2}+4 c_{2}^{2}+Q_{21}-c_{1} Q_{22}\right)^{2}+ \\
& 256 w\left(8 c_{1} c_{2}+Q_{22}\right)\left(8 c_{1}^{2} c_{2}-4 c_{2}^{2}-Q_{21}+c_{1} Q_{22}\right)-256 w^{3}\left(8 c_{1}^{3} c_{2}-c_{1}\left(-4 c_{2}^{2}+Q_{21}\right)\right) \\
& -256 w^{3}\left(c_{1}^{2} Q_{22}+c_{2} Q_{22}\right)+64 w^{2}\left(32 c_{2}^{3}+Q_{22}^{2}+8 c_{2}\left(Q_{21}+c_{1} Q_{22}\right)\right)+ \\
& 76 w^{5}\left(w^{\prime}\right)^{2}+152 w^{4} c_{1}\left(w^{\prime}\right)^{2}+64 w^{3}\left(c_{1}^{2}+3 c_{2}\right)\left(w^{\prime}\right)^{2}-64 w\left(4 c_{2}^{2}+Q_{21}\right)\left(w^{\prime}\right)^{2}- \\
& 64 w^{2}\left(6 c_{1} c_{2}+Q_{22}\right)\left(w^{\prime}\right)^{2}+ \\
& 128\left(8 c_{1}^{3} c_{2}-c_{1}\left(20 c_{2}^{2}+Q_{21}\right)+c_{1}^{2} Q_{22}+2\left(2 Q_{20}-c_{2} Q_{22}\right)\right)\left(w^{\prime}\right)^{2}-20 w^{2}\left(w^{\prime}\right)^{4}-16 w c_{1}\left(w^{\prime}\right)^{4}+ \\
& 16\left(c_{1}^{2}-4 c_{2}\right)\left(w^{\prime}\right)^{4}+80 w^{3}\left(w^{\prime}\right)^{2} w^{\prime \prime}+96 w^{2} c_{1}\left(w^{\prime}\right)^{2} w^{\prime \prime}+128 w c_{2}\left(w^{\prime}\right)^{2} w^{\prime \prime}- \\
& 32\left(8 c_{1} c_{2}+Q_{22}\right)\left(w^{\prime}\right)^{2} w^{\prime \prime}-8\left(w^{\prime}\right)^{4} w^{\prime \prime}-10 w^{4}\left(w^{\prime \prime}\right)^{2}-16 w^{3} c_{1}\left(w^{\prime \prime}\right)^{2}-32 w^{2} c_{2}\left(w^{\prime \prime}\right)^{2}+ \\
& 16 w\left(8 c_{1} c_{2}+Q_{22}\right)\left(w^{\prime \prime}\right)^{2}-32\left(-8 c_{1}^{2} c_{2}+4 c_{2}^{2}+Q_{21}-c_{1} Q_{22}\right)\left(w^{\prime \prime}\right)^{2}+ \\
& \left(20 w+8 c_{1}\right)\left(w^{\prime}\right)^{2}\left(w^{\prime \prime}\right)^{2}+\left(w^{\prime \prime}\right)^{4}=0
\end{aligned}
$$

Two cases when $c=0$, and $c=0, q=0$ give us shorter ODEs:

$c=0$

$$
\begin{aligned}
& 25 w^{8}+160 w^{4} Q_{21}+256 Q_{21}^{2}-80 w^{5} Q_{22}-256 w Q_{21} Q_{22}+64 w^{2} Q_{22}^{2}+ \\
& 76 w^{5}\left(w^{\prime}\right)^{2}+512 Q_{20}\left(w^{\prime}\right)^{2}-64 w Q_{21}\left(w^{\prime}\right)^{2}-64 w^{2} Q_{22}\left(w^{\prime}\right)^{2}-20 w^{2}\left(w^{\prime}\right)^{4}+ \\
& 80 w^{3}\left(w^{\prime}\right)^{2} w^{\prime \prime}-32 Q_{22}\left(w^{\prime}\right)^{2} w^{\prime \prime}-8\left(w^{\prime}\right)^{4} w^{\prime \prime}-10 w^{4}\left(w^{\prime \prime}\right)^{2}-32 Q_{21}\left(w^{\prime \prime}\right)^{2}+ \\
& 16 w Q_{22}\left(w^{\prime \prime}\right)^{2}+20 w\left(w^{\prime}\right)^{2}\left(w^{\prime \prime}\right)^{2}+\left(w^{\prime \prime}\right)^{4}=0
\end{aligned}
$$

$c=0, q=0$

$$
\begin{aligned}
& 25 w^{8}+76 w^{5}\left(w^{\prime}\right)^{2}-20 w^{2}\left(w^{\prime}\right)^{4}+80 w^{3}\left(w^{\prime}\right)^{2} w^{\prime \prime}- \\
& 8\left(w^{\prime}\right)^{4} w^{\prime \prime}-10 w^{4}\left(w^{\prime \prime}\right)^{2}+20 w\left(w^{\prime}\right)^{2}\left(w^{\prime \prime}\right)^{2}+\left(w^{\prime \prime}\right)^{4}=0
\end{aligned}
$$

Later on we shall see that these equations has 2-dimensional commutative symmetry Lie algebra generated by translations and the 1-st KdV, and therefore can be solved in quadratures.

Now we apply theorem (5) to spectral problems for the Shrödinger ordinary differential equations in which potentials are solutions of $n$-th KdV equations. This gives us complete and explicit solutions of the spectral problems.

We illustrate this method for potentials which satisfy the first KdV equation (this is the case of a special Lame equation) and for the following boundary problem on an interval $[a, b]$

$$
y(a)=y(b)=0 .
$$


Then theorem (5) shows that smooth eigen functions

$$
y(x)=2 \sqrt{\frac{\left|S_{1}(x)\right|}{Q_{1}(\lambda)}} \sin \left(\frac{\sqrt{Q_{1}(\lambda)}}{2} \int_{a}^{x} \frac{d \tau}{S_{1}(\tau)}\right)
$$

do exist if:

- $S_{1}=\lambda+\frac{w}{2}+c_{1} \neq 0$ on the interval,

- $Q_{1}(\lambda)>0$,

$$
\int_{a}^{b} \frac{d \tau}{S_{1}(\tau)}=\frac{2 \pi n}{\sqrt{Q_{1}(\lambda)}}
$$

for $n \in \mathbb{Z}$.

Summarizing we get the following result.

Theorem 6 Let potential $w$ satisfies the classical KdV equation, $w^{\prime \prime \prime}+6 w w^{\prime}+$ $4 c_{1} w^{\prime}=0$, then spectral values $\lambda$ for the boundary problem (10) are given by formula

$$
\lambda=\wp\left(\alpha, g_{2}, g_{3}\right)-2 c_{1} / 3
$$

where $\alpha$ are solutions of the equations

$$
2(b-a) \zeta(\alpha)+\ln \frac{\sigma(b+c-\alpha) \sigma(a+c+\alpha)}{\sigma(a+c-\alpha) \sigma(b+c+\alpha)}=2 \pi n i, \quad n \in \mathbb{Z},
$$

such that $Q_{1}(\lambda)>0$ and

$$
\lambda>-c_{1}-\frac{1}{2} \min [w(x), a \leq x \leq b],
$$

or

$$
\lambda<-c_{1}-\frac{1}{2} \max [w(x), a \leq x \leq b] .
$$

Here

$$
Q_{1}(\lambda)=-4 \lambda^{3}-8 c_{1} \lambda^{2}+q_{11}(w) \lambda+q_{10}(w)
$$

and constants $q_{11}(w)$ and $q_{10}(w)$ are values of first integrals $q_{10}$ and $q_{11}$ on the solution $w$. Function $\zeta(\alpha)$ is the Weierstrass zeta function and $\sigma(z)$ is the Weierstrass sigma function with invariants

$$
g_{2}=\frac{4 c_{1}^{2}-6 c_{1}}{3}, g_{3}=-\frac{152 c_{1}^{3}}{27}-\frac{5 q_{11}(w) c_{1}}{3}+2 q_{10}(w) .
$$

The eigen functions corresponding to the eigenvalue $\lambda$ have the following form:

$$
y_{\lambda}(x)=2 \sqrt{\frac{\left|\lambda+\frac{w(x)}{2}+c_{1}\right|}{Q_{1}(\lambda)}} \sin \left(\frac{\sqrt{Q_{1}(\lambda)}}{2}\left(2(x-a) \zeta(\alpha)+\ln \frac{\sigma(x-\alpha) \sigma(a+\alpha)}{\sigma(x+\alpha) \sigma(a-\alpha)}\right)\right)
$$


Proof. Since

$$
w=-2 \wp\left(x+c, g_{2}, g_{3}\right)-\frac{2 c_{1}}{3}
$$

we have

$$
I=\int_{a}^{b} \frac{d \tau}{\lambda+\frac{w}{2}+c_{1}}=\int_{a}^{b} \frac{d \tau}{\lambda-\wp\left(\tau+c, g_{2}, g_{3}\right)+2 c_{1} / 3}
$$

Let $\alpha$ be such that

$$
\wp\left(\alpha, g_{2}, g_{3}\right)=\lambda+2 c_{1} / 3
$$

Then (see [7])

$$
I=-\int_{a+c}^{b+c} \frac{d \tau}{\wp\left(\tau, g_{2}, g_{3}\right)-\wp\left(\alpha, g_{2}, g_{3}\right)}=-\left.\frac{1}{\wp^{\prime}\left(\alpha, g_{2}, g_{3}\right)}\left(2 z \xi(\alpha)+\ln \frac{\sigma(z-\alpha)}{\sigma(z+\alpha)}\right)\right|_{a+c} ^{b+c} .
$$

Because

$$
\wp^{\prime 2}\left(x, g_{2}, g_{3}\right)=4 \wp^{3}\left(x, g_{2}, g_{3}\right)-g_{2} \wp\left(x, g_{2}, g_{3}\right)-g_{3}
$$

and

$$
\wp^{\prime \prime}\left(x, g_{2}, g_{3}\right)=6 \wp^{2}\left(x, g_{2}, g_{3}\right)-g_{2} / 2,
$$

we get the following values of the first integrals $q_{11}$ and $q_{10}$ :

$$
\begin{aligned}
q_{11}(w) & =w^{\prime \prime}+3 w^{2}+4 c_{1} w-4 c_{1}^{2}=g_{2}-16 / 3 c_{1}^{2}=-2 c_{1}-4 c_{1}^{2}, \\
q_{10}(w) & =\frac{2 w w^{\prime \prime}-w^{\prime 2}+4 w^{3}}{4}+c_{1}\left(w^{\prime \prime}+4 w^{2}+4 c_{1} w\right) \\
& =2 / 3 c_{1} g_{2}+g_{3}-32 / 27 c_{1}^{3} .
\end{aligned}
$$

Note that for

$$
\wp\left(\alpha, g_{2}, g_{3}\right)=\lambda+2 c_{1} / 3
$$

we have

$$
\wp^{\prime 2}\left(\alpha, g_{2}, g_{3}\right)=4 \wp^{3}\left(\alpha, g_{2}, g_{3}\right)-g_{2} \wp\left(\alpha, g_{2}, g_{3}\right)-g_{3}=-Q_{1}(\lambda) .
$$

Therefore

$$
\wp^{\prime}\left(\alpha, g_{2}, g_{3}\right)= \pm \sqrt{-Q_{1}(\lambda)}
$$

and we get that

$$
I= \pm\left.\frac{1}{\sqrt{-Q_{1}(\lambda)}}\left(2 z \xi(\alpha)+\ln \frac{\sigma(z-\alpha)}{\sigma(z+\alpha)}\right)\right|_{a+c} ^{b+c}=\frac{2 \pi n}{\sqrt{Q_{1}(\lambda)}} .
$$

So we have

$$
\pm\left.\left(2 z \zeta(\alpha)+\ln \frac{\sigma(z-\alpha)}{\sigma(z+\alpha)}\right)\right|_{a+c} ^{b+c}=2 \pi n i,
$$

where $\zeta(\alpha)$ is the Weierstrass zeta function and $\sigma(z)$ is the Weierstrass sigma function. 
Finally we get the following equations for $\alpha$ :

$$
2(b-a) \zeta(\alpha)+\ln \frac{\sigma(b+c-\alpha) \sigma(a+c+\alpha)}{\sigma(a+c-\alpha) \sigma(b+c+\alpha)}=2 \pi n i .
$$

In the similar way one gets the following result.

Theorem 7 Let potential $w$ satisfies the $n$-th $K d V$ equation, then spectral values $\lambda$ for the boundary problem (10) are given by solutions of the equation

$$
\int_{a}^{b} \frac{d \tau}{S_{n}(\tau)}=\frac{2 \pi m}{\sqrt{Q_{n}(\lambda)}}, \quad m \in \mathbb{Z}
$$

such that $Q_{n}(\lambda)>0$ and $S_{n}(\tau) \neq 0$ on the interval $[a, b]$.

The eigen functions corresponding to the eigenvalue $\lambda$ have the following form:

$$
y_{\lambda}(x)=2 \sqrt{\frac{\left|S_{n}(x)\right|}{Q_{n}(\lambda)}} \sin \left(\frac{\sqrt{Q_{n}(\lambda)}}{2} \int_{a}^{x} \frac{d \tau}{S_{n}(\tau)}\right)
$$

\subsection{Dynamics}

It is common to use symmetries to integrate ordinary differential equations. Now we turn it over and will use ODEs for integration of evolutionary differential equations.

Let

$$
u_{t}=\varphi\left(x, u, u_{x}, \ldots, \frac{\partial^{k} u}{\partial x^{k}}\right)
$$

and we are looking for ordinary differential equations

$$
F\left(x, y, y^{\prime}, y^{\prime \prime}, \ldots, y^{(m-1)}, y^{(m)}\right)=0
$$

such that the Lie equation of this equation satisfies for the given generating function $\varphi$. In other words, we'll find such ODEs that $\phi$ is a generating function for shuffling symmetry of them.

In this case any solution of the Cauchy problem

$$
\left\{\begin{array}{c}
\frac{\partial u}{\partial t}=\varphi\left(x, u, u_{x}, \ldots, \frac{\partial^{m+k} u}{\partial x^{m+k}}\right), \\
\left.u\right|_{t=0}=h_{0}(x)
\end{array}\right.
$$

under the condition that $h_{0}(x)$ satisfies our ordinary differential equation can be found as path $h_{t}$

$$
u(t, x)=h_{t}(x)
$$

in the space of all solutions of the ODE $F\left(x, y, y^{\prime}, y^{\prime \prime}, \ldots, y^{(m-1)}, y^{(m)}\right)=0$ (see 2.2). 
Conditions for $F$ is given by equation (6):

$$
\sum_{i=0}^{m} \frac{\partial F}{\partial p_{i}} \delta^{i}(\widetilde{\varphi})=0, \text { if } F=0 .
$$

In this equation $\widetilde{\varphi}$ is the restriction generating function $\phi$ on the equation $F^{-1}(0)$ and all its prolongations:

$$
\begin{gathered}
F=0, \\
\mathbf{D}(F)=0, \\
\mathbf{D}^{2}(F)=0, \ldots,
\end{gathered}
$$

where

$$
\mathbf{D}=\frac{\partial}{\partial x}+p_{1} \frac{\partial}{\partial p_{0}}+\cdots+p_{m} \frac{\partial}{\partial p_{m-1}}+\cdots
$$

is the total derivative.

We'll consider relation (12) as a differential equation on $F$, and for solutions $F$ of the equation we call ordinary differential equation $F=0$ dynamics of order $m$ for evolutionary equation (11).

Note that in this case to find trajectory of a solution $h(x)$ one should integrate the system

$$
\left\{\begin{array}{c}
\dot{p_{0}}=\widetilde{\varphi} \\
\dot{p_{1}}=\delta(\widetilde{\varphi}), \\
\cdots \cdots \\
\dot{p_{m}}=\delta^{m}(\widetilde{\varphi})
\end{array}\right.
$$

on $\mathcal{E}$ with the initial conditions

$$
\left\{\begin{array}{c}
\left.p_{0}\right|_{t=0}=h(x), \\
\left.p_{1}\right|_{t=0}=h^{\prime}(x), \\
\cdots \cdots \\
\left.p_{m}\right|_{t=0}=h^{(m)}(x) .
\end{array}\right.
$$

Then $p_{0}(t)=h_{t}(x)$ gives the unknown function $h_{t}(x)$, and $u(t, x)=h_{t}(x)$ is a solution of the evolutionary equation $\frac{\partial u}{\partial t}=\varphi\left(x, u, u_{x}, \ldots, \frac{\partial^{k} u}{\partial x^{k}}\right)$.

\section{Dynamics for the $\mathrm{KdV}$ equation}

As an illustration for the method let's investigate finite dimensional dynamics for the $\mathrm{KdV}$ equation:

$$
u_{t}=u \cdot u_{x}+u_{x x x}
$$

Substitution $u=6 w$ establishes the relation between $\mathrm{KdV}$ equations considered above and this equation. We rewrite functions $z_{n}(w), S_{n}(\lambda, w), Q_{n}(\lambda, w)$ in 
the canonical coordinates $\left(p_{0}, \ldots, p_{n}, \ldots\right)$ on the jet spaces where $p_{0}$ corresponds to $u$.

In these notations we have the following Lenard's recursion (see [2]):

$$
z_{0}=1, z_{1}=\frac{p_{0}}{12}+c_{1}, \cdots
$$

and

for $n=0,1, \ldots$. , and

$$
\mathbf{D}\left(z_{n+1}\right)=\frac{1}{4} L\left(z_{n}\right)
$$

$$
L=\mathbf{D}^{3}+\frac{2}{3} p_{0} \mathbf{D}+\frac{1}{3} p_{1}
$$

Functions

$$
K_{n}=\mathbf{D}\left(z_{n+1}\right)
$$

corresponds to $n$-th stationary KdV equations.

Let

$$
S_{n}=\sum_{i=0}^{n} z_{i} \lambda^{n-i}
$$

and

$$
Q_{n}=2 S_{n} \mathbf{D}\left(K_{n-1}\right)+\frac{2}{3} p_{0} S_{n}^{2}-4 \lambda S_{n}^{2}-K_{n-1}^{2}=\sum_{i=0}^{2 n+1} q_{n i} \lambda^{i}
$$

Then we get

$$
X_{\phi}\left(z_{n}\right)=\mathbf{D}^{3}\left(z_{n}\right)+p_{0} z_{n},
$$

and

$$
X_{\phi}\left(K_{n}\right)=\mathbf{D}^{3}\left(K_{n}\right)+p_{1} \mathbf{D}\left(K_{n}\right)+p_{0} K_{n},
$$

where $\phi=p_{3}+p_{0} p_{1}$, and

$$
X_{\phi}=\sum_{i=0} D^{i}(\phi) \frac{\partial}{\partial p_{i}}
$$

This shows that differential equations corresponding to linear combinations of $z_{n}$ or $K_{n}$ give finite dimensional dynamics for the $\mathrm{KdV}$ equation. Moreover, functions $q_{n i}$ also produce finite dimensional dynamics.

Remark that order of $z_{n+1}$ equals $2 n$, and order $K_{n}$ is $2 n+1$. Therefore $z$ 's give even dimensional and $K$ 's odd dimensional dynamics.

Summarizing we arrive to the following result.

Theorem 8 Let $a_{0}, \ldots, a_{n} \in \mathbb{R}$ be constants. Then differential equations:

$$
\begin{aligned}
\sum_{i=0}^{n} a_{i} z_{i} & =0 \\
\sum_{i=0}^{n} a_{i} K_{i} & =0 \\
q_{n i} & =0
\end{aligned}
$$

give finite dimensional dynamics for the $K d V$ equation. 
In addition to the above theorem the low dimensional dynamics given by polynomials can be found by direct computations. Below we give and investigate some of them in dimensions $\leq 3$.

\subsection{1-st order dynamics}

One can check that functions

$$
F=3 p_{1}^{2}+p_{0}^{3}+a_{2} p_{0}^{2}+a_{1} p_{0}+a_{0}
$$

satisfy the equation of dynamic for $\phi=p_{0} p_{1}+p_{3}$ and arbitrary constants $a_{0}, a_{1}, a_{2}$.

The solution space $\mathbf{S o l}(\mathcal{E})$ can be identified with curve

$$
3 p_{1}^{2}+p_{0}^{3}+a_{2} p_{0}^{2}+a_{1} p_{0}+a_{0}=0
$$

on the plane $\left(p_{0}, p_{1}\right)$.

The vector field $X_{\phi}$ on the ODE $\mathcal{E}$ has the following form

$$
-\frac{a_{2}}{3}\left(p_{1} \frac{\partial}{\partial p_{0}}-\frac{a_{1}+2 a_{2} p_{0}+3 p_{0}^{2}}{6} \frac{\partial}{\partial p_{1}}\right) .
$$

Moreover, the vector field

$$
p_{1} \frac{\partial}{\partial p_{0}}-\frac{a_{1}+2 a_{2} p_{0}+3 p_{0}^{2}}{6} \frac{\partial}{\partial p_{1}}
$$

is Hamiltonian with respect to the standard symplectic structure $d p_{1} \wedge d p_{0}$ with Hamiltonian

$$
H=3 p_{1}^{2}+p_{0}^{3}+a_{2} p_{0}^{2}+a_{1} p_{0} .
$$

In other words the curves $H=$ const define the solution spaces and the Hamiltonian flow is the flow generated by KdV. 


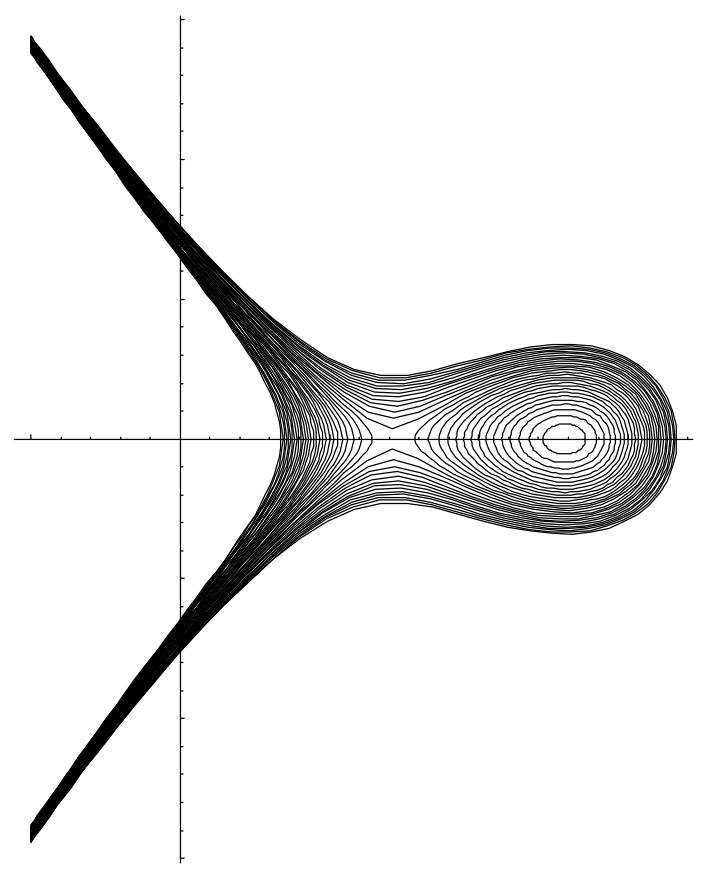

KdV 1-st order dynamics

Solutions of the equation $F=0$, has the form

$$
u=-12 \wp\left(x+c, g_{2}, g_{3}\right)-\frac{a_{2}}{3}
$$

where $\wp\left(x, g_{2}, g_{3}\right)$ is the Weierstrass elliptic function with invariants

$$
g_{2}=\frac{a_{2}^{2}-3 a_{1}}{108}, g_{3}=\frac{27 a_{0}-9 a_{1} a_{2}+2 a_{2}^{3}}{11664} .
$$

The shift of these solutions along $X_{\phi}$ leads us to the following solutions of the $\mathrm{KdV}$ equation

$$
u(x, t)=-12 \wp\left(x-\frac{a_{2} t}{3}+c, g_{2}, g_{3}\right)-\frac{a_{2}}{3} .
$$




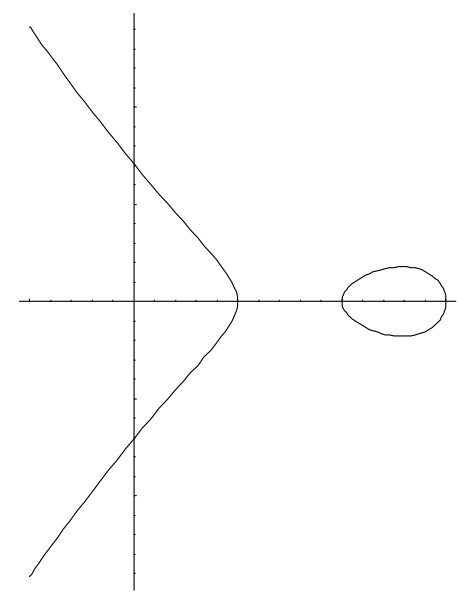

The solution space

These pictures show the solution space and a trajectory for 1-st order dynamics with

$$
F=p_{1}^{3}+\left(p_{0}-1\right)\left(p_{0}-2\right)\left(p_{0}-3\right) .
$$

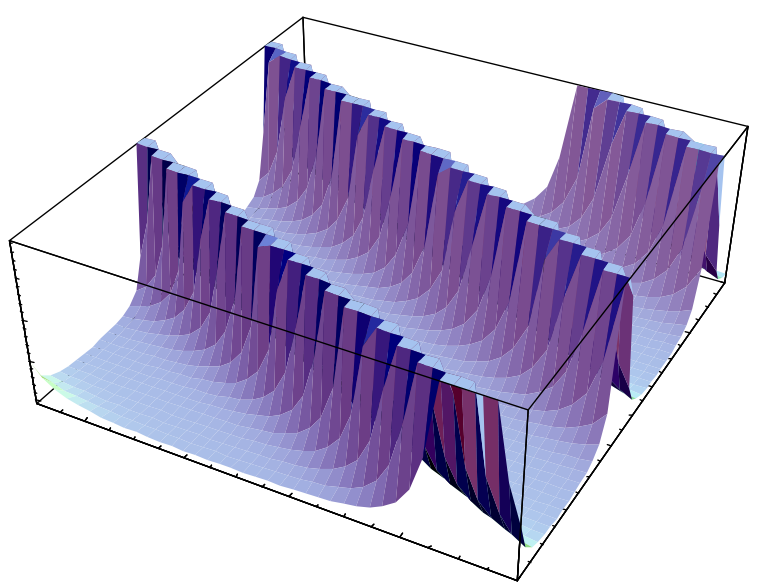

The trajectory

\subsection{Second Order Dynamics}

Here we describe the second order dynamics. We shall consider dynamics $F\left(p_{0}, p_{1}, p_{2}\right)=0$ which are invariant with respect to the scale symmetry $x p_{x}+$ $3 t p_{t}+2 p_{0}$ for $\mathrm{KdV}$ equation. We assign for $p_{0}$ weight $2, p_{1}$ weight 3 and $p_{2}$ weight 4 and assume that $F$ is a sum of homogeneous polynomial of degree $\leq n$ . The following list gives non-trivial homogeneous dynamics for small $n$ :

$n=4$

$$
F_{4}=p_{0}^{2}+2 p_{2}+a p_{0}+b,
$$




$$
\begin{gathered}
n=6 \\
F_{6}=2\left(a+3 p_{0}\right) p_{2}-6 p_{1}^{2}+p_{0}^{3}+3 a p_{0}^{2}+b p_{0}+c, \\
n=8 \quad F_{8}=p_{0}^{4} / 4+p_{0}^{2} p_{2}+p_{2}^{2}+a\left(2 p_{0}^{3}-3 p_{1}^{2}+6 p_{0} p_{2}\right)+b\left(p_{0}^{2}+2 p_{2}\right)+c
\end{gathered}
$$

where $a, b$ and $c$ are constants, and

$n=10$

$$
F_{10}=8 p_{2}^{3}+9 p_{1}^{4} .
$$

Note that dynamics $F_{4}$ and $F_{6}$ coincide with $q_{10}$ and $q_{11}$.

The previous differential equation $\mathcal{E}_{J T}$ gives us the following dynamics:

$$
\begin{aligned}
& 16384 c_{1}^{4} c_{2}^{2}-16384 c_{1}^{2} c_{2}^{3}+4096 c_{2}^{4}+\frac{8192}{3} c_{1}^{3} c_{2}^{2} p_{0}-\frac{4096}{3} c_{1} c_{2}^{3} p_{0}+\frac{512}{9} c_{2}^{3} p_{0}^{2}- \\
& \frac{256}{27} c_{1}^{3} c_{2} p_{0}^{3}-\frac{128}{27} c_{1} c_{2}^{2} p_{0}^{3}-\frac{16}{9} c_{1}^{2} c_{2} p_{0}^{4}+\frac{56}{81} c_{2}^{2} p_{0}^{4}-\frac{4}{81} c_{1} c_{2} p_{0}^{5}+\frac{1}{729} c_{1}^{2} p_{0}^{6}+ \\
& \frac{5 c_{2} p_{0}^{6}}{1458}+\frac{5 c_{1} p_{0}^{7}}{17496}+\frac{25 p_{0}^{8}}{1679616}+\frac{256}{9} c_{1}^{3} c_{2} p_{1}^{2}-\frac{640}{9} c_{1} c_{2}^{2} p_{1}^{2}-\frac{32}{27} c_{2}^{2} p_{0} p_{1}^{2}- \\
& \frac{8}{27} c_{1} c_{2} p_{0}^{2} p_{1}^{2}+\frac{2}{243} c_{1}^{2} p_{0}^{3} p_{1}^{2}+\frac{2}{81} c_{2} p_{0}^{3} p_{1}^{2}+\frac{19 c_{1} p_{0}^{4} p_{1}^{2}}{5832}+\frac{19 p_{0}^{5} p_{1}^{2}}{69984}+\frac{1}{81} c_{1}^{2} p_{1}^{4}- \\
& \frac{4}{81} c_{2} p_{1}^{4}-\frac{1}{486} c_{1} p_{0} p_{1}^{4}-\frac{5 p_{0}^{2} p_{1}^{4}}{11664}-\frac{32}{27} c_{1} c_{2} p_{1}^{2} p_{2}+\frac{8}{81} c_{2} p_{0} p_{1}^{2} p_{2}+\frac{1}{81} c_{1} p_{0}^{2} p_{1}^{2} p_{2}+ \\
& \frac{5 p_{0}^{3} p_{1}^{2} p_{2}}{2916}-\frac{1}{972} p_{1}^{4} p_{2}+\frac{64}{9} c_{1}^{2} c_{2} p_{2}^{2}-\frac{32}{9} c_{2}^{2} p_{2}^{2}+\frac{16}{27} c_{1} c_{2} p_{0} p_{2}^{2}-\frac{2}{81} c_{2} p_{0}^{2} p_{2}^{2}- \\
& \frac{1}{486} c_{1} p_{0}^{3} p_{2}^{2}-\frac{5 p_{0}^{4} p_{2}^{2}}{23328}+\frac{1}{162} c_{1} p_{1}^{2} p_{2}^{2}+\frac{5 p_{0} p_{1}^{2} p_{2}^{2}}{1944}+\frac{p_{2}^{4}}{1296}+\frac{128}{9} p_{1}^{2} Q_{20}- \\
& 4096 c_{1}^{2} c_{2} Q_{21}+2048 c_{2}^{2} Q_{21}-\frac{1024}{3} c_{1} c_{2} p_{0} Q_{21}+\frac{128}{9} c_{2} p_{0}^{2} Q_{21}+\frac{32}{27} c_{1} p_{0}^{3} Q_{21}+ \\
& \frac{10}{81} p_{0}^{4} Q_{21}-\frac{32}{9} c_{1} p_{1}^{2} Q_{21}-\frac{8}{27} p_{0} p_{1}^{2} Q_{21}-\frac{8}{9} p_{2}^{2} Q_{21}+256 Q_{21}^{2}+4096 c_{1}^{3} c_{2} Q_{22}- \\
& 2048 c_{1} c_{2}^{2} Q_{22}+\frac{2048}{3} c_{1}^{2} c_{2} p_{0} Q_{22}-\frac{512}{3} c_{2}^{2} p_{0} Q_{22}+\frac{128}{9} c_{1} c_{2} p_{0}^{2} Q_{22}-\frac{32}{27} c_{1}^{2} p_{0}^{3} Q_{22}- \\
& \frac{32}{27} c_{2} p_{0}^{3} Q_{22}-\frac{2}{9} c_{1} p_{0}^{4} Q_{22}-\frac{5}{486} p_{0}^{5} Q_{22}+\frac{32}{9} c_{1}^{2} p_{1}^{2} Q_{22}-\frac{64}{9} c_{2} p_{1}^{2} Q_{22}- \\
& \frac{4}{81} p_{0}^{2} p_{1}^{2} Q_{22}-\frac{4}{27} p_{1}^{2} p_{2} Q_{22}+\frac{8}{9} c_{1} p_{2}^{2} Q_{22}+\frac{2}{27} p_{0} p_{2}^{2} Q_{22}-512 c_{1} Q_{21} Q_{22}- \\
& \frac{128}{3} p_{0} Q_{21} Q_{22}+256 c_{1}^{2} Q_{22}^{2}+\frac{128}{3} c_{1} p_{0} Q_{22}^{2}+\frac{16}{9} p_{0}^{2} Q_{22}^{2}=0
\end{aligned}
$$

Let's look at the dynamics in more details.

3.2.1 $\quad F_{4}=p_{0}^{2}+2 p_{2}+a p_{0}+b$

In this case vector field $-\frac{2}{a} X_{\phi}$ is the restriction of the vector field

$$
V_{\phi}=p_{1} \frac{\partial}{\partial p_{0}}+p_{2} \frac{\partial}{\partial p_{1}}-\frac{\left(a+2 p_{0}\right) p_{1}}{2} \frac{\partial}{\partial p_{2}}
$$


on the zero level $F_{4}=0$, and $X_{\phi}$ can be integrated in the same way as for 1-st order dynamics.

Trajectories of $X_{\phi}$ are given by formulae

$$
p_{0}(t)=-12 \wp\left(t+K, g_{2}, g_{3}\right)-a / 2
$$

where

$$
g_{2}=\frac{a^{2}-12 a b}{48}, g_{3}=\frac{a^{3}-12 a b-12 c^{2}}{1738}
$$

and the constant can be found from initial data.

These formulas lead us to the following pathes in the solution space

$$
u(t, x)=-12 \wp\left(t-\frac{a x}{2}+\text { const }, g_{2}, g_{3}\right)-a / 2
$$

with arbitrary invariant $g_{3}$ and $g_{2}$ given above.

3.2.2 $\quad F_{6}=2\left(a+3 p_{0}\right) p_{2}-6 p_{1}^{2}+p_{0}^{3}+a p_{0}^{2}+b p_{0}+c$

Differential equation $\mathcal{E}=F_{6}^{-1}(0)$ has singular points at

$$
\begin{aligned}
& p_{0}=-\frac{a}{3}, \\
& p_{1}^{2}=\frac{a^{3}}{81}-\frac{a b}{18}+\frac{c}{6} .
\end{aligned}
$$

Moreover, in this case symmetries $X_{p_{1}}$ and $X_{\phi}$ are linear dependent on the differential equation $\mathcal{E}$ and

$$
X_{\phi}=-H\left(p_{1} \frac{\partial}{\partial p_{0}}+\frac{6 p_{1}^{2}-p_{0}^{3}-a p_{0}^{2}-b p_{0}-c}{2\left(a+3 p_{0}\right)} \frac{\partial}{\partial p_{1}}\right),
$$

where

$$
H=\frac{a b+3 c+6 b p_{0}-6 p_{0}^{3}-18 p_{1}^{2}}{2\left(a+3 p_{0}\right)^{2}}
$$

is the fist integral for $\mathcal{E}$, and for vector filed $X_{\phi}$ :

$$
X_{\phi}(H)=0
$$

on $\mathcal{E}$.

Vector field $X_{\phi}$ has also singularities at the points where $a+3 p_{0} \neq 0$, and

$$
p_{0}^{3}+a p_{0}^{2}+b p_{0}+c=0, \quad p_{1}=0 .
$$

Depending on roots of polynomial $p_{0}^{3}+a p_{0}^{2}+b p_{0}+c$ we have the following three types of phase portraits:

- Three real distinct roots. In the following picture we take roots: $-1,1,2$, and

$$
F_{6}=2\left(3 p_{0}-2\right) p_{2}-6 p_{1}^{2}+p_{0}^{3}-2 p_{0}^{2}-p_{0}+2
$$




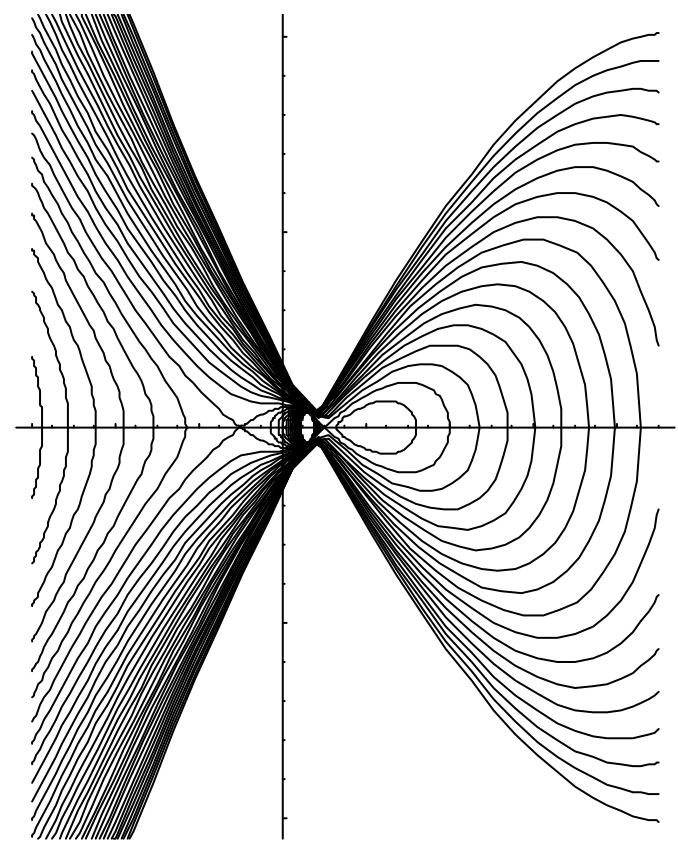

- A real root of multiplicity 2. In the picture we take roots: $1,1,2$, and $F_{6}=2\left(3 p_{0}+4\right) p_{2}-6 p_{1}^{2}+p_{0}^{3}-4 p_{0}^{2}+5 p_{0}-2$ 


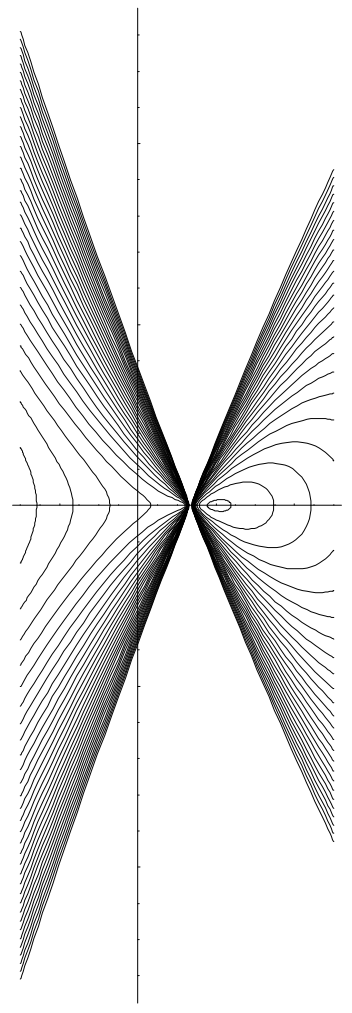

- Two complex roots. In the picture we take roots: $1, \frac{-1+\sqrt{-3}}{2}, \frac{-1-\sqrt{-3}}{2}$, and

$$
F_{61}=6 p_{0} p_{2}-6 p_{1}^{2}+p_{0}^{3}-1
$$




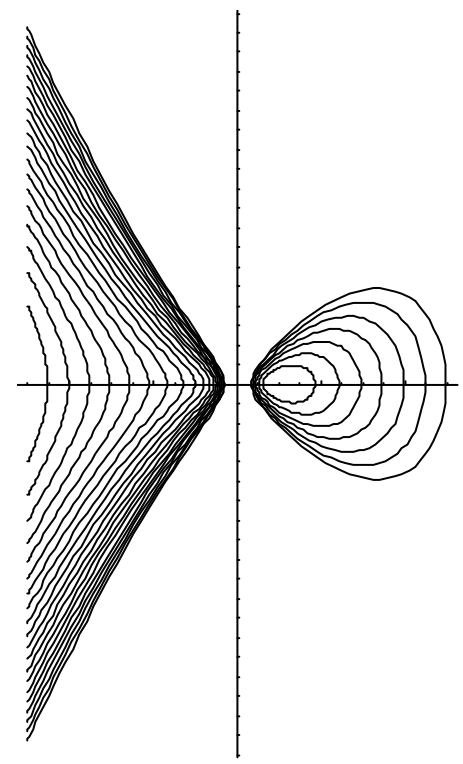

Solutions of the equation $F_{6}^{-1}(0)$ one can find from the first integral $H$. So they are solutions of the following 1-st order ODE

$$
p_{1}^{2}=-\frac{p_{0}^{3}}{3}-k p_{0}^{2}+\frac{b-2 a k}{3} p_{0}+\frac{a b+3 c-2 a^{2} k}{18}
$$

for some constant $k, H=k$.

Thus solutions of the ODE can be represented in terms of the Weierstrass function as follows

$$
u(x)=-12 \wp\left(x+C_{0}, g_{2}, g\right)-k
$$

where

$$
\begin{aligned}
& g_{2}=\frac{k(b+k-2 a k)}{12}, \\
& g_{3}=\frac{12 k^{3}-12 a k^{2}+2\left(a^{2}+3 b\right) k-a b-c}{2592} .
\end{aligned}
$$

Note that along $X_{\phi}$ function $H$ is constant and $X_{\phi}=-H X_{p_{1}}$. Therefore the corresponding path in the solution space is

$$
u(x, t)=-12 \wp\left(x-k t+C_{0}, g_{2}, g_{3}\right)-k .
$$

3.3 $F=6\left(p_{0}-\lambda\right) p_{2}-6 p_{1}^{2}+\left(p_{0}-\lambda\right)^{3}$

This the special case when the polynomial $p_{0}^{3}+a p_{0}^{2}+b p_{0}+c$ has root $\lambda$ of multiplicity 3 . Without loss of generality we can assume that $\lambda=0$, and investigate the ordinary differential equation $\mathcal{E}$, where

$$
F=6 p_{0} p_{2}-6 p_{1}^{2}+p_{0}^{3}=0 .
$$


Vector field $X_{\phi}$ on $\mathcal{E}$ is proportional to $X_{p_{1}}$

$$
X_{\phi}=-H X_{p_{1}}
$$

with

$$
H=-\frac{p_{0}^{3}+3 p_{1}^{2}}{3 p_{0}^{2}}
$$

Where $H$ is a first integral for ordinary differential equation $\mathcal{E}$ and for the vector field $X_{\phi}$.

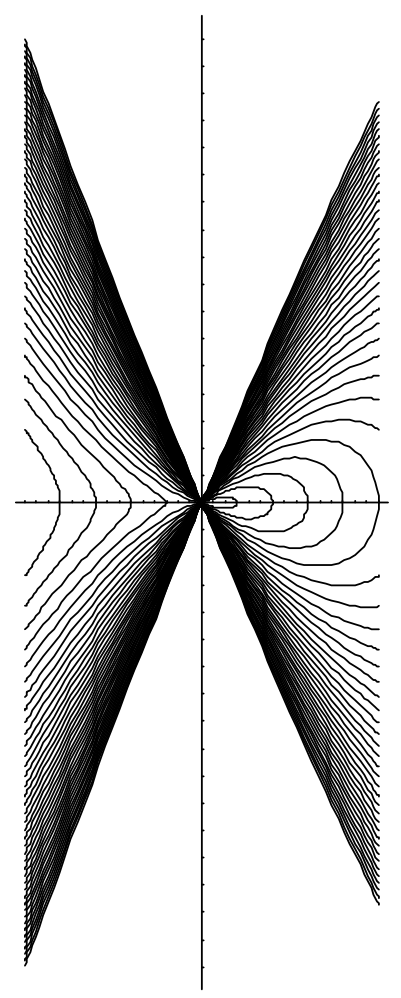

The ODE $\mathcal{E}$ can be solved directly and one gets

$$
u(x)=\frac{a^{2}}{\cosh ^{2}\left(\frac{a(x+b)}{2 \sqrt{3}}\right)} .
$$

Restriction of $H$ on these solutions gives $-a^{2} / 3$, and therefore the corresponding path is the solitary wave solution

$$
u(x, t)=\frac{a^{2}}{\cosh ^{2}\left(\frac{a\left(x+a^{2} t / 3+b\right)}{2 \sqrt{3}}\right)} .
$$




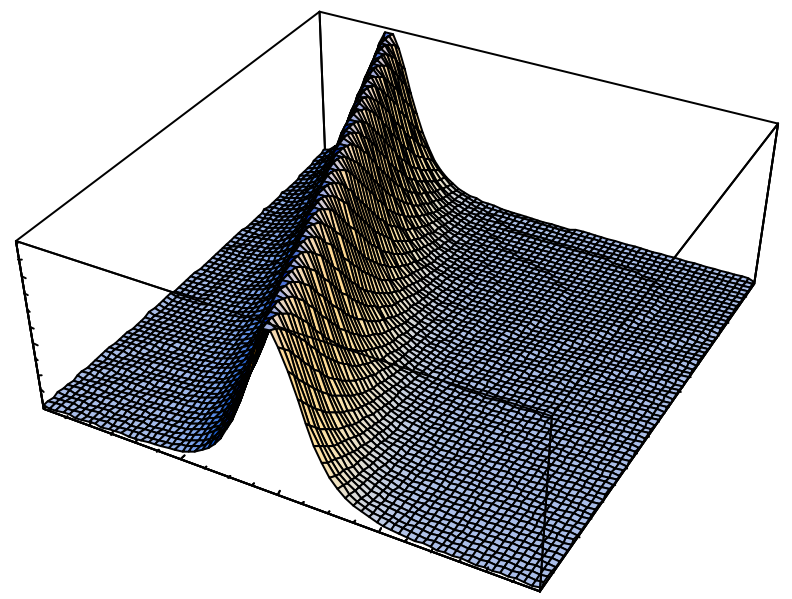

3.3.1 $\quad F_{10}=8 p_{2}^{3}+9 p_{1}^{4}$

In this case the vector field $X_{\phi}$ on $F_{10}^{-1}(0)$ is proportional $X_{p_{1}}$ :

$$
X_{\phi}=-H X_{p_{1}}
$$

with

$$
H=\frac{3 p_{1}^{2}-2 p_{0} p_{2}}{2 p_{2}} .
$$

Solutions of the equation $F_{10}=0$ has the form

$$
u(x)=B-\frac{12}{(x+A)^{2}},
$$

and $H=-B$ for these solutions. Therefore the corresponding path has the form of the rational solution

$$
u(x, t)=B-\frac{12}{(x+A-B t)^{2}} .
$$

\subsubsection{Trivial dynamics}

The following ODEs

$$
\begin{aligned}
& 2 p_{0}^{3}-3 p_{1}^{2}+6 p_{0} p_{2}+a\left(p_{0}^{2}+2 p_{2}\right)+b, \\
& p_{0}^{4} / 4+p_{0}^{2} p_{2}+p_{2}^{2}+a\left(2 p_{0}^{3}-3 p_{1}^{2}+6 p_{0} p_{2}\right)+b\left(p_{0}^{2}+2 p_{2}\right)+c, \\
& p_{0}^{4} / 4+p_{0}^{2} p_{2}+p_{2}^{2}+a\left(2 p_{0}^{3}-3 p_{1}^{2}+6 p_{0} p_{2}\right)\left(p_{0}^{2}+2 p_{2}\right)+c
\end{aligned}
$$

give the trivial dynamics: $X_{\phi}=0$ on $\mathcal{E}$-s. 


\subsection{Third Order Dynamics}

The following dynamics represent non trivial polynomial dynamics in degree $\leq 10$ :

$$
\begin{aligned}
& F_{1}=a p_{1}+b\left(p_{0} p_{1}+p_{3}\right)+\frac{1}{2} p_{0}^{2} p_{1}-p_{1} p_{2}+p_{0} p_{3} \\
& F_{2}=p_{3}^{2}+2 p_{1}^{2} p_{2}-a\left(p_{3}+p_{0} p_{1}\right)^{2} \\
& F_{3}=\left(p_{3}+p_{1} p_{0}+a\right)\left(p_{1}+b p_{0}+c\right)
\end{aligned}
$$

where $a, b, c$ are constants.

\subsubsection{Dynamics for $F_{1}=a p_{1}+b\left(p_{0} p_{1}+p_{3}\right)+\frac{1}{2} p_{0}^{2} p_{1}-p_{1} p_{2}+p_{0} p_{3}$}

In this case $X_{\phi}=H X_{p_{1}}$, where

$$
H=\frac{2 p_{2}+p_{0}^{2}-2 a}{2\left(b+p_{0}\right)}
$$

is a first integral of ordinary differential equation $F_{1}^{-1}(0)$.

Solutions of ordinary differential equations $H=c$, where $c$ is a constant, or

$$
p_{2}=-\frac{p_{0}^{2}}{2}+c p_{0}+a+b c
$$

can be expressed in terms of the Weierstrass functions:

$$
u=-12 \wp\left(x+c_{1}, g_{2}, g_{3}\right)-c
$$

with arbitrary $g_{3}$ and

$$
g_{2}=\frac{(1-3 b) c^{2}-3 a c}{12} .
$$

The corresponding pathes in the solution space are

$$
u(x, t)=-12 \wp\left(x+c t+c_{1}, g_{2}, g_{3}\right)-c .
$$

3.4.2 Dynamics for $F_{2}=p_{3}^{2}+2 p_{1}^{2} p_{2}-a\left(p_{3}+p_{0} p_{1}\right)^{2}$

Let $a \neq 1$, then $X_{\phi}=H X_{p_{1}}$, where

$$
H=\frac{p_{0} \pm \sqrt{a p_{0}^{2}+2(1-a) p_{2}}}{1-a}
$$

is a first integral of ordinary differential equation $F_{2}^{-1}(0)$.

Solutions of ordinary differential equations $H=c$, where $c$ is a constant,

$$
p_{2}=\frac{p_{0}^{2}}{2}-c p_{0}+c^{2}(1-a)
$$


can be expressed in terms of the Weierstrass functions:

$$
u=12 \wp\left(x+c_{1}, g_{2}, g_{3}\right)+c
$$

with arbitrary $g_{3}$ and

$$
g_{2}=\frac{c^{2}}{12}+\frac{c^{3}(1-a)}{2} .
$$

The corresponding pathes in the solution space are

$$
u(x, t)=12 \wp\left(x+c t+c_{1}, g_{2}, g_{3}\right)+c .
$$

In the case $a=1$, we have

$$
F_{2}=p_{1}^{2}\left(2 p_{2}-p_{0}^{2}\right)-2 p_{0} p_{1} p_{3}
$$

and $X_{\phi}=H X_{p_{1}}$, where

$$
H=\frac{p_{0}^{2}+2 p_{2}}{2 p_{0}}
$$

is a first integral of ODE $F_{2}^{-1}(0)$.

Solutions of ODEs $H=c$, where $c$ is a constant,

$$
p_{2}=-\frac{p_{0}^{2}}{2}+c p_{0}
$$

can be expressed in terms of the Weierstrass functions:

$$
u=-12 \wp\left(x+c_{1}, g_{2}, g_{3}\right)-c
$$

with arbitrary $g_{3}$ and

$$
g_{2}=\frac{c^{2}}{12} .
$$

The corresponding pathes in the solution space are

$$
u(x, t)=-12 \wp\left(x+c t+c_{1}, g_{2}, g_{3}\right)-c .
$$

\subsection{Fourth order dynamics}

Fourth order dynamics are defined by functions

$$
F=p_{4}+\frac{5 p_{0} p_{2}}{3}+\frac{5 p_{1}^{2}}{6}+\frac{5 p_{0}^{3}}{18}+a\left(p_{2}+\frac{p_{0}^{2}}{2}\right)+b p_{0}+c
$$

where $a, b, c$ are constants.

It is easy to check that the vector field $X_{\phi}$ and the ODE $F^{-1}(0)$ has the following first integral

$$
H_{1}=-36 b p_{0}^{2}-12 a p_{0}^{3}-5 p_{0}^{4}-12 p_{0}\left(6 c+5 p_{1}^{2}\right)+36\left(6 a c-a p_{1}^{2}+p_{2}^{2}-2 p_{1} p_{3}\right),
$$


and the restriction on $H_{1}=k$ admits first integral

$$
\begin{gathered}
H_{2}=72\left(2 a^{2}+5 b\right) p_{0}^{6}+120 a p_{0}^{7}+25 p_{0}^{8}+24 p_{0}^{5}\left(36 a b+30 c+19 p_{1}^{2}\right)+432 p_{1}^{4}\left(3 a^{2}-12 b-4 p_{2}\right) \\
+2 p_{0}^{4}\left(648 b^{2}-216 a c+5 k+684 a p_{1}^{2}-180 p_{2}^{2}\right)+ \\
\left(-216 a c+k-36 p_{2}^{2}\right)^{2}-24 p_{0}^{3}\left(216\left(a^{2}-b\right) c-a k+36 a p_{2}^{2}-12 p_{1}^{2}\left(3 a^{2}+9 b+10 p_{2}\right)\right) \\
-216 p_{1}^{2}\left(72\left(a^{2}+2 b\right) c-a k-12 p_{2}\left(4 c+a p_{2}\right)\right)- \\
72 p_{0}^{2}\left(72(3 a b-c) c-b k+10 p_{1}^{4}+36 b p_{2}^{2}-12 p_{1}^{2}\left(3 a b+4 c+6 a p_{2}\right)\right) \\
+24 p_{0}\left(-36 a p_{1}^{4}+6 c\left(-216 a c+k-36 p_{2}^{2}\right)+p_{1}^{2}\left(432 a c-k+36 p_{2}\left(12 b+5 p_{2}\right)\right)\right) .
\end{gathered}
$$

The last ordinary differential equation $H_{2}=k_{2}$ has two symmetries $X_{\phi}$ and $X_{p_{1}}$ and they are independent and commute. Therefore the differential equation can be integrated in quadratures.

Namely, the method discussed above gives two 1-forms

$$
\begin{aligned}
\theta_{0} & =\frac{p_{2} d p_{0}-p_{1} d p_{1}}{G}, \\
\theta_{1} & =\frac{A d p_{0}+B d p_{1}}{G}-d x,
\end{aligned}
$$

where

$$
\begin{aligned}
G & =c p_{1}+b p_{0} p_{1}+\frac{1}{2} a p_{0}^{2} p_{1}+\frac{5}{18} p_{0}^{3} p_{1}-\frac{p_{1}^{3}}{6}+\frac{3 a c p_{2}}{p_{1}}-\frac{k p_{2}}{72 p_{1}}-\frac{c p_{0} p_{2}}{p_{1}}-\frac{b p_{0}^{2} p_{2}}{2 p_{1}} \\
& -\frac{a p_{0}^{3} p_{2}}{6 p_{1}}-\frac{5 p_{0}^{4} p_{2}}{72 p_{1}}+\frac{1}{2} a p_{1} p_{2}+\frac{5}{6} p_{0} p_{1} p_{2}+\frac{p_{2}^{3}}{2 p_{1}}, \\
A & =c+b p_{0}+a\left(p_{2}+\frac{p_{0}^{2}}{2}\right)+\frac{1}{18}\left(5 p_{0}^{3}-3 p_{1}^{2}+12 p_{0} p_{2}\right), \\
B & =\frac{3 a c}{p_{1}}-\frac{k}{72 p_{1}}-\frac{c p_{0}}{p_{1}}-\frac{b p_{0}^{2}}{2 p_{1}}-\frac{a p_{0}^{3}}{6 p_{1}}-\frac{5 p_{0}^{4}}{72 p_{1}}-\frac{a p_{1}}{2}+\frac{p_{0} p_{1}}{6}+\frac{p_{2}^{2}}{2 p_{1}},
\end{aligned}
$$

and integrals $I_{0}\left(p_{0}, p_{1}\right), I_{1}\left(p_{0}, p_{1}\right)$ such that

$$
\begin{aligned}
& d I_{0}=\frac{p_{2}}{G} d p_{0}-\frac{p_{1}}{G} d p_{1}, \\
& d I_{1}=\frac{A}{G} d p_{0}+\frac{B}{G} d p_{1},
\end{aligned}
$$

and solutions can be found from

$$
I_{0}=c_{0}, I_{1}=x+c_{1}
$$

for some constants $c_{0}, c_{1}$.

\section{References}

[1] Duzhin S.V., Lychagin V.V. Symmetries of distributions and quadrature of ordinary differential equations, Acta Appl. Math. 24,(1991), no.1, p.29-57 
[2] Ibragimov Nail H., Transformation groups in mathematical physics, M. Nauka, 1983

[3] Lax P.D. Integrals of nonlinearequations of evolution and solitary waves. Comm. Pure and Appl. Math., 1968, v.21, p. 467-490

[4] Gardner C.S. Greene J.M., Kruskal M.D., Miura R.M., Kortweg- de Vries equation and generalizations, VI, Methods for exact solution, Comm. Pure and Appl. Math., 1974, v.27, p. 97-133

[5] Krasilshchik I.S., Lychagin V.V., Vinogradov A.M., Geometry of jet spaces and non-linear differential equations, Gordon and Breach, 1986

[6] Kruglikov Boris, Lychagin Valentin, Mayer brackets and solvability PDEs, 1 , Diff. Geometry and Appl., 17 (2002), p.251-272

[7] Abramowitz M., Stegun I.A., Handbook of Mathematical Functions, Dover, 1972 